\title{
RIESGO POTENCIAL POR CAMBIO CLIMÁTICO Y VECTORES. FACTORES LOCALES DE CIUDAD: USO DE SUELO Y VEGETACIÓN
}

\author{
Carlos Manuel WELSH-RODRIGUEZ ${ }^{1}$, Carolina Andrea OCHOA-MARTINEZ ${ }^{1}$, \\ Marco Aurelio MORALES-MARTINEZ ${ }^{1}$, María Esther NAVA-BRINGAS ${ }^{1}$, \\ Q'enty DELGADO ${ }^{1}$, Andrew MONAGHAN ${ }^{2}$, Mary HAYDEN ${ }^{2}$, \\ Ramos HERRERA-ZAVALETA ${ }^{1}$ \\ ${ }^{1}$ Centro de Ciencias de la Tierra. Universidad Veracruzana. \\ ${ }^{2} R A L$. National Center for Atmospheric Research. \\ cwelsh@uv.mx
}

\section{RESUMEN}

El cambio climático está relacionado con la presencia y abundancia de vectores, en particular con Aedes aegypti (responsable de la trasmisión de dengue, chikungunya y zika) a nivel urbano; nuestra hipótesis asocia la presencia del vector con la climatología y factores socio-ambientales tales como el uso de suelo y la vegetación en espacios urbanos, mismos que inciden en favorecer un hábitat para el vector. Estudiamos una región tropical en la parte central del golfo de México desde los 0 y hasta los 2200 m.s.n.m., ya que es un reto observar el acoplamiento entre los sistemas mencionados para estimar un posible escenario de riesgo presente y futuro ante el calentamiento del sistema climático. Se usan datos de la investigación de 2011 a 2014, donde se ha estudiado la presencia de Aedes aegypti en un transecto altitudinal, con datos de factores urbanos para observar el acoplamiento entre dichas variables por medios estadísticos.

Palabras clave: Riesgo, hábitat, cambio climático, vector.

\begin{abstract}
The climatic change is related with the vector presence and abundance, particularly with Aedes aegypti (responsible of dengue, chikungunya and zika transmission) at urban level; our hypothesis associate vector presence with socio-environmental factors and climatology, as land use and vegetation in urban areas, these provide easily a vector habitat. We studied a tropical region on the central Gulf of México from 0 to 2200 meters above sea level, due to the challenge of coupled with the factors mentioned before, in order to estimate a possible risk scenario present or future associated at global warming. We use research data from 2011 to 2014, in which we studied the presence of Aedes on altitude transect, with urban data to observe trough statistical methods variables coupling.
\end{abstract}

Key words: Risk, habitat, climatic change, vector

\section{INTRODUCCIÓN}

Según una estimación reciente de casos de dengue, se producen 390 millones de infecciones cada año (intervalo creíble del 95\%: 284 a 528 millones), de los cuales 
96 millones (67 a 136 millones) se manifiestan clínicamente (cualquiera que sea la gravedad de la enfermedad) (Bhatt S. et al., 2013 en OMS, 2016). En otro estudio sobre la prevalencia del dengue se estima que 3900 millones de personas, de 128 países, están en riesgo de infección por los virus del dengue (Brady O.J., 2012 en OMS, 2016), enfermedad transmitida por el vector Aedes aegypti y para la cual no hay vacuna. El aumento acelerado de casos en América, llevo a un grupo de instituciones: Universidad Veracruzana (UV), Colorado State University (CSU) y el National Center for Atmospheric Research (NCAR) a desarrollar un proyecto de investigación de 2011 a 2014 donde se ha estudiado la presencia y abundancia de Aedes aegypti en un transecto altitudinal del nivel del mar hasta los 2100 m.s.n.m., financiado por la National Science Foundation (NSF)

Uno de los hallazgos más significativos fue confirmar las cotas de presencia del vector y su relación positiva con variables climáticas, puesto que no había sido reportada la presencia del vector por encima de los 1800 m.s.n.m. y se encontró el vector entre los 1700 y 2130 m.s.n.m., fueron pocos individuos pero suficientes para poder confirmar que ya alcanzaron dicha cota (Lozano Fuentes et al., 2012a).

Con la finalidad de integrar productos en la base de datos SERVIR, Crosson et al., (2012) emplearon datos de percepción remota (NASA), utilizando observaciones ambientales tales como clima, uso de suelo, tipo de vegetación, humedad y la presencia/abundancia del vector. Como resultado de lo anterior los autores estimaron un modelo de regresión lineal donde se encontró el vector, asociado a variables tales como precipitación, temperatura y se exploró de manera muy general el uso de suelo.

Mientras que Moreno et al., (2014) utilizando sensores remotos, evaluaron la abundancia del vector con las condiciones climáticas en la zona de montaña superior a los 1700 m.s.n.m., donde se encontraron correlaciones fuertes entre la abundancia del mosquito, la temperatura de superficie y la precipitación, situación que dejó establecer la hipótesis de la posible relación con factores socio-ambientales locales como uso de suelo y vegetación que dependen de la gestión y ordenamiento municipal.

\subsection{Región de estudio}

El Valle de Orizaba es una región amplia en la zona montañosa central del estado de Veracruz, sitio que conecta el corredor de comercio más grande de la República Mexicana entre el Golfo de México (teniendo como punto de partida la ciudad y Puerto de Veracruz) y llegando a la ciudad de México. El estudio está focalizado en las ciudades de Orizaba, Camerino Z. Mendoza, Maltrata y Acultzingo, entre altitudes que van desde los 1300 m.s.n.m. hasta 2100 m.s.n.m. es una zona con topografía muy variada con condiciones geográficas que inciden en una abundante precipitación en verano con escurrimientos y vegetación abundante. La ciudad de Puebla será utilizada como sitio de control debido a las condiciones climatológicas y topográficas.

Esta región es conocida por su riqueza vegetal, la cual está compuesta por selva mediana subperennifolia, bosque de encino y bosque mesófilo de montaña en la ciudad de Orizaba. La ciudad de Mendoza tiene presencia de bosque de pinos, encinos, coníferas en la zona de mayor altitud y en las de menor cuenta con vegetación de galería como ahuehuetes y álamos principalmente. Por su parte las ciudades de Maltrata 
y Acultzingo comparten mayormente similitudes en su vegetación la cual es bosque de pináceas (Florescano y Ortiz, 2010).

En conjunto las ciudades cuentan con una población total de 200,644 habitantes al 2010 en cifras de Instituto Nacional de Estadística Geografía e Informática (INEGI). Las actividades económicas de estas ciudades son diversas y cubren los tres sectores productivos, por su parte, las ciudades de Orizaba y Mendoza tienen un sector terciario desarrollado el cual cubre el $75 \%$ y $71 \%$ de su demanda de empleos respectivamente, las ciudades de Maltrata y Acultzingo cuentan con un sector terciario menos desarrollado, no obstante este es el de mayor ocupación de empleos con el $46.1 \%$ y $41.3 \%$ respectivamente, en estas dos últimas ciudades el sector primario toma fuerza, ocupando el $23.2 \%$ y $28.2 \%$ de la fuerza laboral (SEFIPLAN, 2015).

\section{TRANSECTO DE ESTUDIO Veracruz - Puebla}
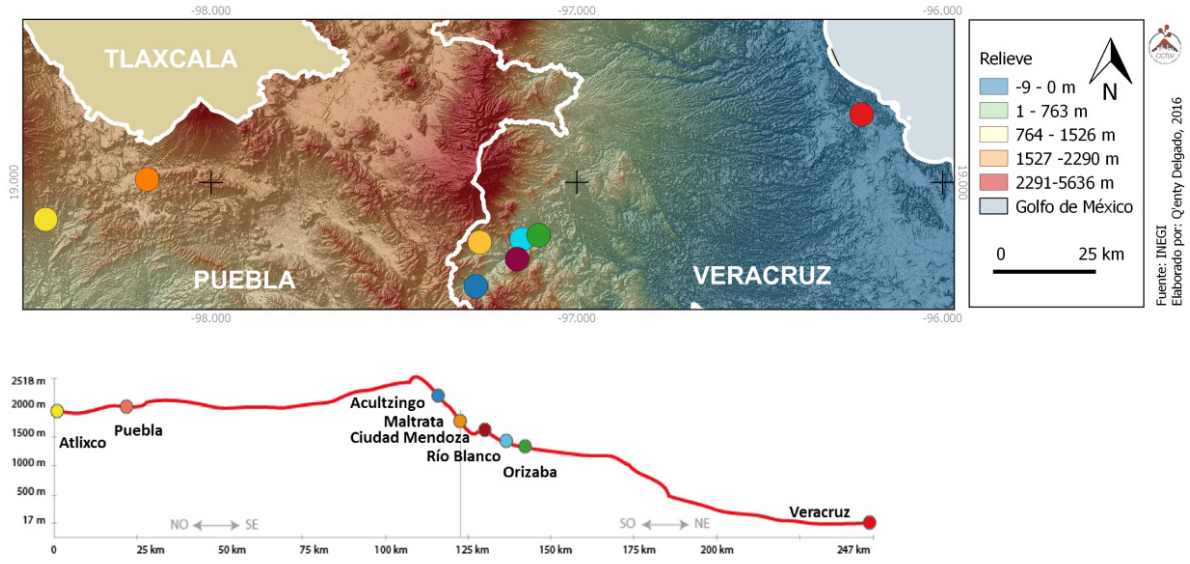

Fig. 1: Transecto de estudio original.

\subsection{El clima local}

Tiene diversos microclimas, que van desde el semicálido húmedo y el templado húmedo, ambos con lluvias abundantes en verano en las ciudades de Orizaba y Mendoza, hasta el templado subhúmedo y en ciudades como Acultzingo y Maltrata.

De acuerdo con Ruíz et al., (2010), la zona presenta una temperatura media anual entre los 16 y $18^{\circ} \mathrm{C}$ (una precipitación entre los 1000 y 1600 milímetros de lluvia anuales). A continuación se describen los meses representativos para cada estación en la zona de estudio: Para el caso de la temperatura mínima promedio Enero y Octubre presentan $6.9^{\circ} \mathrm{C}$; Abril y Julio tienen $11.7^{\circ} \mathrm{C}$; mientras que la temperatura máxima promedio es de Enero $21^{\circ} \mathrm{C}$, Abril $26.9^{\circ} \mathrm{C}$, Julio $23.7^{\circ} \mathrm{C}$ y Octubre $23.4^{\circ} \mathrm{C}$. Así también se muestra que Puebla es la ciudad con las temperaturas mínimas más bajas en los 80's, mientras las siguientes ciudades presentan las temperaturas mínimas más bajas en la década de los 2000 . 


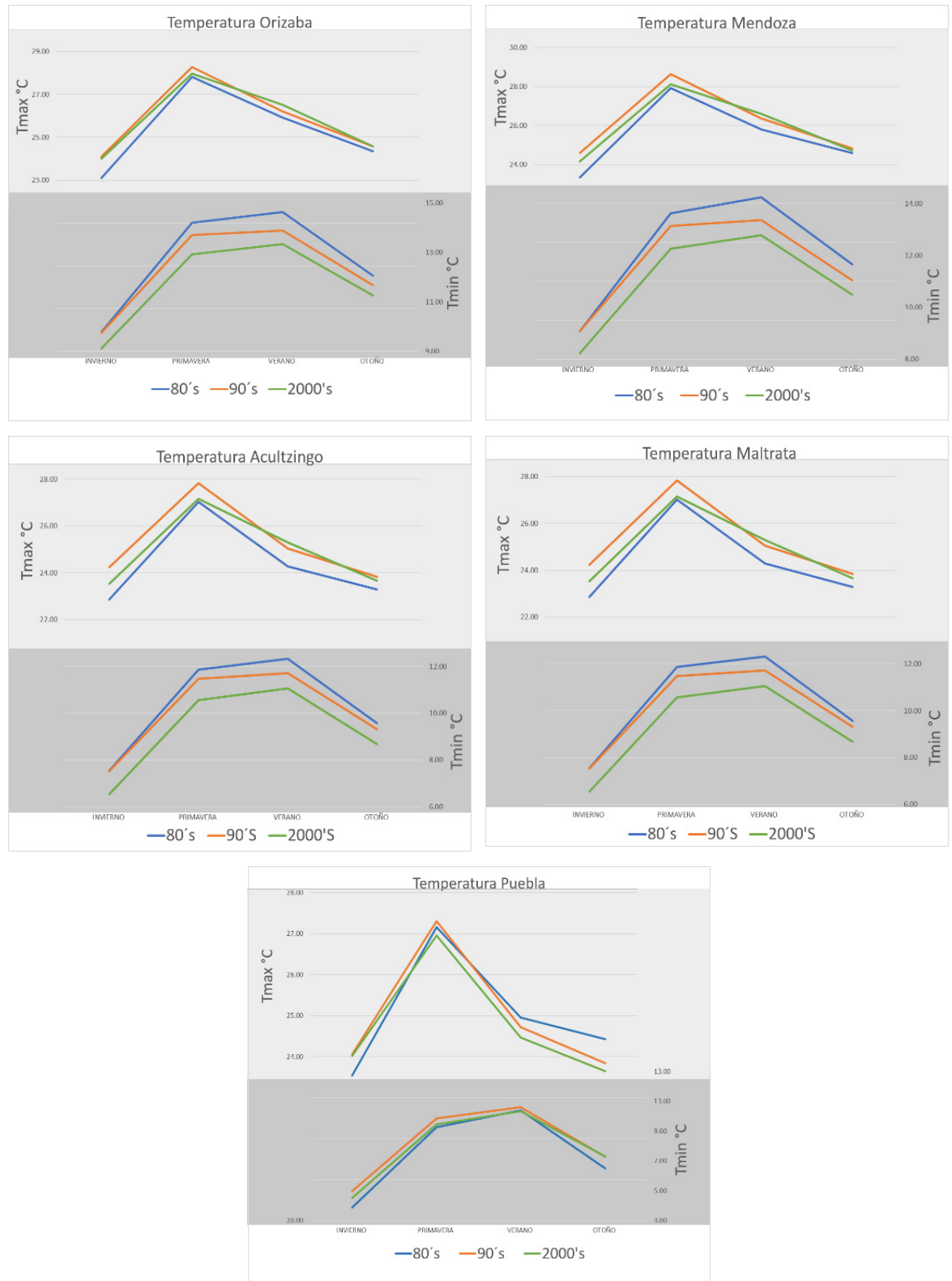

Fig. 2: Variables climatológicas en la región de estudio. Fuente:NASA,2016.

\subsection{El uso de suelo}

El uso de suelo es una variable compleja por múltiples razones, la falta de datos locales, las clasificaciones que utilizan diferentes categorías en función de la vocación municipal y contar con datos históricos. En virtud de lo anterior y con las imágenes 
disponibles $L A N D S A T$ se realizó una reclasificación en nueve clases: área agrícola, cuerpos de agua, pastizal, bosque, selva, vegetación baja, zona urbana, sin vegetación y vegetación hidrófila.

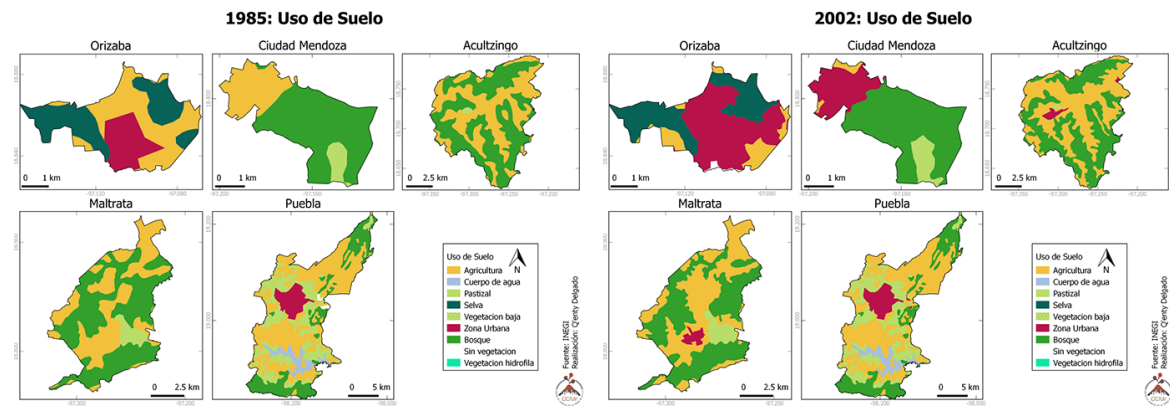

Fig. 3: Uso de suelo en la región de estudio. Fuente: INEGI Serie I y II uso de suelo modificado, Landsat 1985 y 2002.

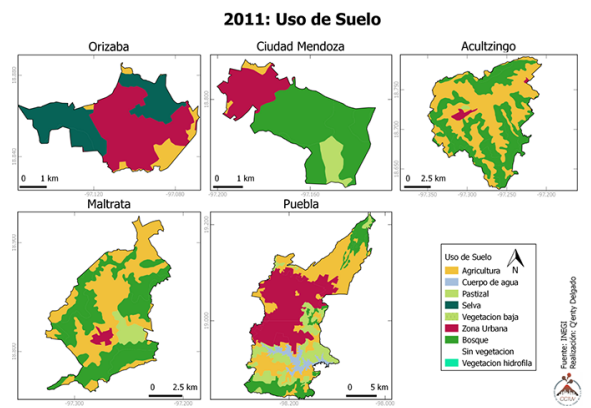

Fig.4: Uso de suelo en la región de estudio. Fuente: INEGI Serie V uso de suelo modificado, Landsat2011.

\subsection{La vegetación local}

La vegetación local cobra importancia por la relación directa al favorecer un hábitat propicio para la presencia y abundancia del vector, sobre todo si han existido cambios bruscos en el marco temporal de cuatro décadas que han traído cambios significativos en el entorno. Carrington et al., (2013), demostraron que las condiciones fluctuantes de temperatura alteraban de manera significativa el ciclo de vida del mosquito vector. De la misma forma, Chowell et al., (2011) demostraron que el dengue persiste en áreas con vegetación de tipo selvática y clima tropical mostrando su pico más alto durante la temporada de lluvia y con ello pudieron determinar que las epidemias de dengue se presentan de manera cíclica lo cual está fuertemente asociado a los ciclos de temperaturas estacionales en la región del Perú.

\subsubsection{Orizaba}

Se encuentran tres tipos principales de vegetación original que son: la selva mediana subperennifolia, bosque de encino y bosque mesófilo de montaña. Además de 
estos tipos de vegetación, también hay vegetación secundaria derivados de estos, cuyas especies pertenecen en su mayoría a las familias Compositae, Gramineae, Leguminosae y Euphorbiaceae. En el Cerro del Borrego dos tipos de vegetación: bosque mesófilo de montaña y selva mediana subperennifolia, montaña y selva mediana subperennifolia. Esta diversidad vegetal arbustiva y arbórea permite la captación de humedad en el ambiente circundante y la mantiene a lo largo del año, disminuyendo la temperatura durante la temporada de lluvia.

\subsubsection{Camerino Z. Mendoza}

Su ubicación geográfica permite la presencia de diversos ecosistemas como bosque de pinos, encinos, coníferas en la parte alta y hacia la parte baja se caracteriza por tener una vegetación de galería por la presencia de ahuehuetes y álamos principalmente, los cuales sirven de refugio a una diversidad importante de flora y fauna. El tipo de vegetación de éste municipio permite la generación de sombra y con ello mantiene la temperatura y humedad ambiental de la zona.

\subsubsection{Maltrata}

Los ecosistemas que coexisten en el municipio son los de bosque frío de pinaceas con pino colorado, ayacahuite, encino, cedro, fresno, tecojote, capulín y ocote. En el municipio se cuenta con recursos forestales en los cuales encontramos las siguientes especies: pino colorado, pinos estrobus, ayacacuite, encino, tecojote, capulín y ocote. La ubicación geográfica de Maltrata permite la conservación de la humedad ambiental lo cual está favorecido por el tipo de vegetación circundante de la zona.

\subsubsection{Acultzingo}

Los ecosistemas que coexisten en el municipio son el de bosque de pináceas. Su riqueza está representada por su vegetación en donde sobresalen el pino y el encino por lo apreciado de su madera. Cabe mencionar que este tipo de ecosistemas fríos son bien estudiados por contribuir a la captación de humedad ambiental.

\subsubsection{Puebla}

La ciudad tiene especies introducidas en los años 20's y 40's como palmeras, eucaliptos, álamos y jacarandas. El único árbol endémico del estado es el Cuatecomate (Crescentia alata), el cual llega a medir entre cinco y 14 metros de altura y sus flores son amarillo-verdosas y rojas. En este caso, la vegetación original de la zona se ha visto modificada drásticamente por la introducción de especies no nativas, las cuales si bien no contribuyen a la captación de humedad, sí la conservan al haber actualmente más espacios con sombra en la ciudad y sus alrededores.

\section{MÉTODOS}

Se realizó una climatología básica a partir de datos diarios de reprocesamiento del DAYMET (Daily Surface weather and climatological summaries) de la NASA (National Aeronautics and Space Administration) de los Estados Unidos de Norte América, correspondientes a las décadas de los ochenta, noventa y la primera del 
siglo XXI, además de los datos tomados in situ del proyecto original, se obtuvieron comportamientos, tendencias y promedios de temperatura y precipitación.

Para el análisis de suelo se realizó una reinterpretación en un Sistema de Información Geográfica (SIG) de imágenes del INEGI, los datos utilizados están a una escala 1/250 000 y las clasificaciones incluidas fueron analizadas y agrupadas de forma que tuvieran sentido en nueve clases para poder comparar cinco años distintos $(1985,1993,2002,2007,2011)$. Con el fin de poder evaluar la posible relación entre el cambio de uso de suelo, el cambio en la vegetación original y urbana, las variables climatológicas se realizaron tres pruebas descriptivas para valorar la dependencia, es decir, que tanto influye una variable sobre la otra; la correlación con la finalidad de cuantificar y medir el alcance de la relación existente entre las variables y el comportamiento.

A partir de imágenes satelitales Landsat se realizó un enfoque sobre el transecto de estudio con énfasis en la mancha urbana para entender la dinámica de la presencia de vegetación in situ (1987 a 2015). A través de una clasificación supervisada por máximo de verosimilitud apoyándose de la composición de falso color de cada imagen, se realizaron tres clases para diferenciar el agua, la vegetación, el suelo sin vegetación con el programa con un SIG. Las clasificaciones fueron supervisadas con muestras de pixeles que se comprobaron con el índice de Kappa para asegurar sus calidades. Como una estrategia para observar la población potencial a partir de datos climatológicos en las décadas señaladas se aplicó el modelo desarrollado por Focks et al., (1993) mismo que permite observar la dinámica de la población de Aedes aegypti denominado Skeeter Buster.

\section{RESULTADOS}

Este apartado se presenta en dos grandes áreas, la primera dedicada al clima urbano, en función de la climatología 1980-2010 y la segunda en relación al hábitat.

\subsection{Clima}

Para la ciudad de Orizaba el análisis de temperatura muestra que la variabilidad en el invierno se ha hecho menor lo que puede incidir en la conservación huevos del vector, por otro lado los otoños han sido más cálidos factor que incide de manera directa en la abundancia del vector, mientras que la temperatura mínima inhibe el ciclo de vida del vector de manera directa, en promedio se observa un ligero incremento que puede estar asociado al cambio en el uso de suelo.

La ciudad que experimentó un cambio radical fue Camerino Z. Mendoza, donde se observa que la anomalía en temperaturas mínimas es cercana a los $2^{\circ} \mathrm{C}$, mientras que la variabilidad se ha incrementado del orden de $3^{\circ} \mathrm{C}$, que va por encima de lo que reporta la literatura a nivel global $(0.1 \%$ década) en poco más del doble, además de observarse un incremento cercano al $20 \%$ en la precipitación de Verano, factores que inciden en favorecer la presencia del vector en la zona, situación que puede ser validada con el incremento de casos confirmados del padecimiento.

Para Maltrata ubicada ligeramente al Norte del transecto con clima templado húmedo y vegetación mixta entre zona templada y fría, se observa que en relación a 
los años $80^{\prime}$ s se ha incrementado la variabilidad en la zona en poco más de $2^{\circ} \mathrm{C}$, con temperaturas mínimas cercanas a $\operatorname{los} 8^{\circ} \mathrm{C}$ en el otoño y $10^{\circ} \mathrm{C}$ para el verano, efecto que puede ser positivo en la presencia del vector en la zona aunado a un incremento mínimo en la precipitación, combinación de factores que pueden ser la razón de los hallazgos de Lozano (Lozano-Fuentes et al., 2012 b).

Acultzingo situado a unos 10 kilómetros en línea recta de Maltrata, donde en el estudio previo se habían encontrado diferencias significativas con Maltrata, se puede observar una tendencia en el incremento de la temperatura máxima para 30 años menor a $1^{\circ} \mathrm{C}$ y un incremento en la precipitación, sin embargo la evidencia de presencia del vector es menor que en Maltrata, situación que puede ser explicada por el grado de conservación del hábitat. En la región de Puebla se tiene que la variabilidad de temperatura se ha incrementado a una tasa diferente a la que ha crecido su infraestructura urbana y el cambio en el uso de suelo, sin embargo es posible observar que hay inviernos más calientes y veranos más lluviosos, además de un incremento en las temperaturas mínimas.

\subsection{Hábitat}

Entre 1985 y 2011 la tasa de crecimiento urbano es superior al 30\% lo que sin lugar duda trajo como consecuencia impactos directos sobre el hábitat potencial del vector. En todos los municipios de estudio, la mancha urbana crece. En unos municipios con vocación rural en 1985, vemos aparecer infraestructura urbana que desplaza vegetación local, por ejemplo en Camerino Z. Mendoza la zona urbana llega ocupar 22,8\% del territorio municipal en 2011. Así mismo, el municipio de Acultzingo ve aparecer una zona urbana después de 1995.

El espacio ocupado por las manchas urbanas de 2011 eran zonas rurales o de vegetación local que incidían en el clima local, tales como espacios agrícolas o pastizales. Entre 1985 y 2011, los bosques tuvieron una ligera reducción cercana al 5\%, la reducción más drástica fue en agricultura y el pastizal. Para el caso de Puebla, vemos que el bosque aumentó en 3\% aproximadamente y el equipamiento urbano estuvo asociado a un crecimiento de espacio verde inducido de casi $25 \%$ lo que incide de manera clara en el clima local de la ciudad.

Los cambios de uso de suelo en el transecto de la zona de estudio entre $1987 \mathrm{y}$ 2015 muestran un cambio significativo en la vegetación dentro de la zona urbana. Comparando los años, se observan cambios drásticos en todos los municipios y de menor intensidad en Acultzingo y Maltrata. La agricultura es el uso que perdió más espacio (Orizaba, Ciudad Mendoza, Río Blanco, Puebla), mismo que fue reemplazado por zonas urbanas. Los municipios de Orizaba, Aculzingo y Matrata, desarrollan una zona urbana desde 1993. Los bosques se redujeron en los municipios centrales del transecto y en Atlixco por manchas reducidas con la presión agrícola o urbana.

\section{DISCUSIÓN}

El clima tiene un rol vital en las enfermedades causadas por vectores. Mismos que son extremadamente sensibles a los efectos directos de temperatura, patrones de precipitación y humedad, ya que influyen en su ciclo de vida. Si el cambio climático 
mejora la longevidad, aumenta la reproducción, aumenta la frecuencia de piquetes de estos insectos a la población o altera sus rangos de distribución, puede ocurrir un aumento en la cantidad de gente infectada (Ize, 2007).

El aumento en el calor en zonas montañosas urbanas es posiblemente la razón del incremento en las infecciones transmitidas por vectores, así mismo los fenómenos extremos tales como inundaciones y sequías favorecen hábitats para el desarrollo de insectos.

\begin{tabular}{|c|c|c|c|c|c|}
\hline Ciudades & $\begin{array}{c}\text { Pob (hab) } \\
\text { INEGI 2010 }\end{array}$ & $\begin{array}{c}\text { Elev. } \\
\text { m.s.n.m. }\end{array}$ & $\begin{array}{c}\text { Uso de } \\
\text { suelo (\% de } \\
\text { cambio) }\end{array}$ & $\begin{array}{c}\text { Var. de } \\
\text { Temp } \\
\left({ }^{\circ} \mathbf{C}, \mathbf{8 0 - 0 0}\right)\end{array}$ & $\begin{array}{c}\text { Aedes } \\
\text { aegypti (\%) }\end{array}$ \\
\hline Orizaba & 121,000 & 1230 & 25 & 13.9 & 0.39 \\
\hline Río Blanco & 40,000 & 1300 & 22.8 & 9.3 & 0.62 \\
\hline C. Mendoza & 35,000 & 1340 & 35 & 9.1 & 0.43 \\
\hline Acultzingo & 7,040 & 1660 & 10 & 9.2 & 0.26 \\
\hline Maltrata & 11,840 & 1797 & 2.86 & -1.5 & 0.07 \\
\hline $\begin{array}{c}\text { Ciudad de } \\
\text { Puebla }\end{array}$ & $1,434,000$ & 2140 & 25 & 13.9 & 0.05 \\
\hline
\end{tabular}

Tabla 1: Factores a evaluar por ciudad.

Los efectos potenciales del cambio de la estructura urbana inciden en el clima local, y si a esto se agrega los efectos cada vez adversos del cambio climático se estarían generando las condiciones ambientales (suficientes o necesarias) para la presencia del vector en sitios donde las condiciones ambientales antes no lo eran, tal como está ocurriendo en la región de estudio, donde se observa que existe una correlación más fuerte entre la presencia del vector con el cambio en el uso de suelo que con la variabilidad de la temperatura.

En estadística, el coeficiente de correlación es utilizado para medir la fuerza y dirección de una relación lineal entre dos variables, siendo usado con mayor frecuencia del Coeficiente de Pearson. La tabla 2 muestra los valores obtenidos para este caso, donde se analizaron la relación Temperatura vs Suelo; Suelo vs Aedes y Temperatura vs Aedes. Se observa que Temperatura vs Suelo y vs Aedes tienen una relación lineal moderada con valores de 0.69 y 0.68 , mientras que la relación existente entre el tipo de suelo y el vector se considera una relación lineal fuerte con un valor de 0.72 . Esta tendencia se mantiene al calcular el coeficiente de determinación y el coeficiente de determinación ajustado.

Sin embargo, si se analizan los errores típicos la relación temperatura vs suelo, tiene el error más grande, lo que debilita la relación; mientras que la relación Temperatura vs Aedes se vuelve estadísticamente más fuertes al tener el error más pequeño.

Ya se había reportado por Lozano Fuentes et al., (2012a) que en México no había sido registrada la presencia del vector por encima de la cota de 1700 m.s.n.m debido a que las condiciones geográficas (climatológicas y de uso de suelo) eran una barrera natural para el vector, sin embargo con los datos modelados en el skeeter buster para 
las décadas previas y con los datos de las capturas e identificación in situ en el verano para el proyecto base, dejan abierta la hipótesis de que la intervención antrópica en zonas de montaña con inviernos menos fríos, tal como se observa en la región de estudio, parece que favorecerá la presencia del vector en un futuro cercano.

\begin{tabular}{|c|c|c|c|}
\hline Análisis & $\begin{array}{c}\text { Temp vs } \\
\text { Suelo }\end{array}$ & $\begin{array}{c}\text { Suelo vs } \\
\text { Aedes }\end{array}$ & Temp vs Aedes \\
\hline Coeficiente de correlación múltiple & 0.69 & 0.72 & 0.68 \\
\hline Coeficiente de determinación $\mathrm{R}^{\wedge} 2$ & 0.48 & 0.53 & 0.47 \\
\hline $\mathrm{R}^{\wedge} 2$ ajustado & 0.30 & 0.37 & 0.29 \\
\hline Error típico & 10.57 & 10.05 & 4.81 \\
\hline
\end{tabular}

Tabla 2: Correlaciones estadísticas obtenidas.

La variabilidad climática y los eventos extremos (sequía e inundación) incidirán en la presencia y abundancia del vector, sabemos que una vez que se pasa la sierra madre oriental hay climas secos y fríos que inciden en el ciclo del vida del vector, sin embargo al estar ligado a una ruta de comercio muy importante el riesgo de contagio es latente ante la presencia de personas portadoras del virus de zonas endémicas, sobre todo cuando en el mismo transecto se localizan ciudades como Camerino Z. Mendoza (1340 ms.n.m.) con una presencia significativa y Alcultzingo (1660 m.s.n.m) donde el vector ya se encuentra establecido en una proporción menor, inclusive en la misma ciudad de puebla se localizaron algunos pocos ejemplares de vector.

Nuestros resultados sirven para señalar que los cambios en políticas públicas asociadas a la conservación y restauración vegetal pueden incidir en favorecer hábitats para la presencia de especies que favorecen el riesgo potencial de adquirir alguna enfermedad como dengue, chikungunya o zika.

\section{AGRADECIMIENTOS}

Es necesario agradecer a la Coordinación de Observatorios de Metropolitanos de la Universidad Veracruzana por el apoyo para la obtención de imágenes y cartografía.

\section{REFERENCIAS}

Bhatt S., Gething P.W., Brady O.J., Messina J.P., Farlow A.W., Moyes C.L., Drake J.M., Brownstein J.S., Hoen A.G., Sankoh O., Myers M.F., Goerge D.B., Jaenisch T., Wint G.R., Simmons C.p., Scott T.W. Farrar J.J., Hay S.I. (2013). The global distribution and burden of dengue. Nature. 496 (7446), 504-507. Doi:10.1038/ nature12060. Epub 2013 Apr 7. Recuperado de http:/www.ncbi.nlm.nih.gov/ pmc/articles/PMC3651993/.

Brady O.J., Gething P.W., Bhatt S., Messina J.P., Brownstein J.S., Hoen A.G., Moyes C.L., Farlow A.W., Scott T.W., Hay S.I. (2012). Refining the global spatial limits of dengue virus transmission by evidence-based consensus. PLoS Negl Trop Dis. 6(8):e1760. doi:10.1371/journal.pntd.0001760. Epub 2012 Aug 7. Recuperado de http://www.ncbi.nlm.nih.gov/pubmed/22880140. 
Carrington L.B., Armijos M.V., Lambrechts L., Barler C.M., Scott T.W. (2013). Effects of fluctuating daily temperatures at critical thermal extremes on Aedes aegypti Life-History traits. PLoS ONE 8(3): e58824. Doi: 10.1371/journal.pone.0058824.

Chowell G., Cazelles B., Broutin H., Munayco C. (2011). The influence of geographic and climate factors on the timing of dengue epidemics in Perú, 1994-2008. BMC Infectious Diseases. 11(164). Doi: 10.1186/1471-2334-11-164. Recuperado de http://www.biomedcentral.com/1471-2334/11/164/prepub.

Crosson, W, Estes, S., Estes, M., Moreno, M., Hemmings, S., Irwing, D., Hayden, M., M. Monaghan., A. Sternhoff, D., Zielinski, E., Welsh, C., Lozano, S., Eisen, L. (2012). Investigation the potential Range Expansion of the vector Mosquito Aedes aegypti in México with NASA Earth Science Remote Sensing Results. Health and Aire Quality Applications Program Review. Newport. RI.

Eastman J.R. (2012). IDRISI SELVA. Guide to GIS and Image Processing. Volume 2. Clark Labs, Clark University. Release 2. 322 p.

Florescano E. y Ortiz J. (Coords). (2010). Atlas del Patrimonio Natural, Histórico y Cultural de Veracruz. Comisión del Estado de Veracruz para la Conmemoración de la Independencia Nacional y de la Revolución Mexicana. México Gobierno del Estado de Veracruz.

Frost Attaway D. and All. (2016). Risk analysis for dengue suitability in Africa using the ArcGIS predictive analysis tools (PA tools), Acta Tropica 158. 248-257.

Focks, D.A., D.G. Haile, E. Daniels, and G.A. Mount. (1993). Dynamic Life Table Model for Aedes aegypti (Diptera: Culicidae): Analysis of the Literature and Model Development. Journal of Medical Entomology 30(6): 1003 - 1015.

Girard (M.C.), GIRARD (C.M.) (2010). Traitement des données de Télédétection, Environnement et Ressources Naturelles, Paris, Dunod, 576 p.

http://www.cuentame.inegi.org.mx/monografias/informacion/pue/territorio/recursos naturales.aspx? tema $=$ me $\& \mathrm{e}=21$

http://www.inafed.gob.mx/work/enciclopedia/EMM30veracruz/municipios/30099a.html http://www.inafed.gob.mx/work/enciclopedia/EMM30veracruz/municipios/30006a.html http://www.mendoza.gob.mx/municipio.html

http://regiondeorizaba.blogspot.mx/2011/07/tipo-de-vegetacion-de-la-zona-de.html http://www.verarboles.com/ZOrizabaZongolica/orizabazongolica.html

Instituto Nacional de Estadística, Geografía e Informática (INEGI). (2010). Censo de Población y Vivienda 2010. Recuperado de http://www.inegi.org.mx/est/ contenidos/proyectos/ccpv/cpv2010/Default.aspx

Ize, I. (2007). Cambio climático y salud humana. INE. México. Recuperado en: http:// www2.ine.gob.mx/publicaciones/gacetas/367/cambioysalud.html 2012.

Lozano-Fuentes S., Hayden M., Welsh-Rodríguez C.M., Ochoa-Martínez C.A., TapiaSantos B., Kobylinski K.C., Uejio C.K., Zielinski-Gutiérrez E., Delle-Monache L., Monaghan A.J., Steinhoff D.F., Eisen L. (2012a). The dengue virus mosquito vector Aedes aegypti at high elevation in México. American Journal of Tropical Medicine and Hygiene. 87(5). 902-909. 
Lozano-Fuentes S., Welsh-Rodríguez C.M., Hayden M., Tapia-Santos B., OchoaMartínez C.A., Kobylinski K.C., Uejio C.K., Zielinski-Gutiérrez E., DelleMonache L., Monaghan A.J., Steinhoff D.F., Eisen L. (2012b). Aedes (Ochlerotatus) epactius along an elevation and climate gradient in Veracruz and Puebla states, México. Journal of Medical Entomology. 49(6). 1244-1253.

Moreno, M., Crosson, W., Eisen, L., Estes, S., Estes, M., Hayden, M., Hemmings, S., Lozano, S., Monaghan, A., Quattrochi, D., Welsh, C. and Zielinski, E. (2014). Correlating Remote Sensing Data with the Abundance of Pupae of the Dengue Virus Mosquito Vector Aedes aegypti, in Central Mexico. SPRS International Journal of Geo-Information. Vol. 3, 732-749. ISSN 2220-9964; doi:10.3390/ ijgi3020732.

NASA (National Aeronautics and Space Administration). (2016). Daily surface weather and climatological summaries.Recuperado de https://daymet.ornl.gov/

Organización Mundial de la Salud (OMS). (2016). Centro de Prensa. Dengue y dengue grave. Nota descriptiva. Abril. Recuperado de http://www.who.int/mediacentre/ factsheets/fs117/es/.

Robin M. , (2002). La télédétection, Nathan Université, 318 p.

Ruiz, A., Tejeda, A., Miranda, S., y Flores, R. H. (2010). Climatología. En G. BenítezBadillo, \& C. Welsh-Rodríguez (Eds.), Atlas del patrimonio natural, histórico y cultural de Veracruz. Tomo I, Patrimonio Natural. (págs. 65-84). Xalapa, México: Gobierno del Estado y Universidad Veracruzana.

Secretaría de Finanzas y Planeación del Estado de Veracruz (SEFIPLAN). (2015). Sistema de Información municipal, Cuadernillos municipales, 2015: Maltrata. Orizaba. Acultzingo. Camerino Z. Mendoza. Gobierno del Estado de Veracruz. 